\title{
Variations of the Microcirculation and It's Fluctua- tion Spectra Changies by the Acupuncture Therapy
}

(Variation of the Fluctuation Spectrum №. 3 )

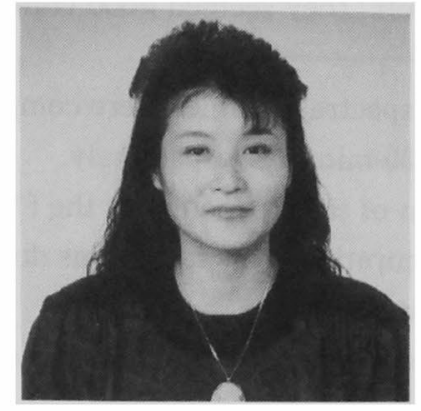

\author{
Mieko Nagumo, \\ Masayuki Fujita, \\ Fukashi Makimoto* \\ Tadahiko Mizuno**
}

\section{(Introduction)}

Many attempts have been done to objectify the acupuncture therapy effect on a patient. The thermographic method is very useful to know the variation of blood flow by the acupuncture. It was considered that the increase of microcirculation means the effective therapy had been given on a patient. In fact, if an effective therapy had been given on a patient, the blood flow increase was observed.

However, at a same time there are few exceptions that some patients showed blood flow decrease in spite of his pain was relieved off.

This means that the method has not completed yet to know the effect of therapy on a patient.

The purporse of this study was to obtainnig a method of the objective decision to the therapy effect on a patient.

We have found the spectrum intensity of blood flow fluctuation was almost in proportion with the inverse of the frequency for the control group without pain or disease ${ }^{(1)(2)}$. This means that the spectrum form is $1 / \mathrm{f}$.

This form is well known as a pink spectrum form by this shape. But also we have found the spectra of the patients who have some pain or disease were far from the pink type which were the white or the brown spectrum.

Figure 1 shows three types of these spectrum : white, pink, and brown respectively. Usually, our fluctuation spectrum such as a blood flow variation is nearly the pink ${ }^{(3)}$. Of course, in this case, it shold be assumed that we have no pain or disease.

\section{(Experimental technics)}

Figure 2 shows the measurement systems. A laser blood flow meter (LBFM) ${ }^{(1)(6)}$ was used 
to measure the blood flow change. Laser light pass through a glass fiber tube and strikes the skin surface which diameter is $1 \mathrm{~mm}$. It goes into skin about $1 \mathrm{~mm}$ depth. It is scattered by redcorpuscle. It goes back through the same fiber tube and is measured by a photo-multitube in the LBFM.

The fluctuation spectrum was calcurated by a micro-computer using the maximum entropy method (MEM).

\section{(Results)}

Figure 3 shows a change of the blood flow for a patient during the therapy. The therapy was given to 50 patients. In all cases, the pain were relieved off ; they seemed good condition after the therapy.

Blood flow showed big variation during the therapy. The spectra changies were compared between before and after therapy : each periods were 20 to 30 minutes respectively.

The spectrum form was shown by an exponential function of the frequency as the $\mathrm{f}^{-\mathrm{n}}$. The frequency range was taken between $10^{-3}$ to $10^{-2} \mathrm{~Hz}$ for the calcuration. This range was decided by the data sampling time and total period of the measurement.

The exponent numbers of fluctuation for the control group is shown in fig. 4 : it shows that all of them are very close to one.

The exponent numbers for the patient group before the therapy shows a big variation as in fig. 5 . However they were approached closer to 1 after the therapy.

The blood flow changies of the patients after the therapy which were compared by the value of the before therapy were shown in fig. 6 . Twenty percents in the patients shwed significant increases of blood flow. No relationships are seen between blood flow change and the spectrum change.

\section{(Discussion)}

Blood flow is changing even if a human is under a rest : fluctuation spectrum shows as $\mathrm{f}^{-1}$ type. The average value that variation spectrum has $\mathrm{f}^{-1}$, would not converge a certain value ; it is impossible to know the effect of therapy on a patient from his blood flow changies.

If the $\mathrm{f}^{-1}$ spectrum is the essential fact to a human being who is comfort: if he has some pain or disease, his spectrum should show some deviation from the $\mathrm{f}^{-1}$.

\section{(Conclusion)}

(1) The spectrum to a human without pain or disease shows $\mathrm{f}^{-1}$.

(2) The spectrum to a patient with some pain or disease shows some deviations from $\mathrm{f}^{-1}$.

(3) The spectrum to a patient after therapy was approached closer to $\mathrm{f}^{-1}$; this means that patients would be relieved off pain.

(4) This spectrum change measurement method seems very useful to the objective decision for the effect on a patient by the therapy.

Nagumo Acupuncture Clinic, Siroishi-ku Sakaedori 17 - 11 - 15, Sapporo city, Japan

* Akiyama Memorial Hospital, Ishikawa-cho 41 - 9, Hakodate city, Japan

** Faculty of Engineering, Hokkaido University, Kita-ku Kita 13 Nishi 8, Sapporo city, Japan 


\section{(References)}

(1) M. Nagumo, M. Fujita and T. Mizuno : J. J. Ryodoraku Medicine, vol. 32, №. 6 - 7 (1987) 18

(2) M. Nagumo, F. Makimoto and T. Mizuno: J. J. Oriental Medicine, vol 34, №. 4 (1988) 106

(3) T. Mushya : J. J. Appl. Phys., vol, 54, №. 5 (1985) 429

(4) H. Fujii, T. Asakura, K. Nohira, Y. shintomi and T. Ohura : Opt. Lett., 10 (1985) 104

(5) H. Fujii, K. Nohira, Y. Shintomi and T. Ohura : 34 th Spring Meeting, Japan Soc. Appl. Phy., 34, №. 3, (1987) 625

(6) H. Fujii, K. Nohira, Y. Yamamoto and T. Ohura : Appl. Opt. vol 26 (1987) 5321

Fig. 1
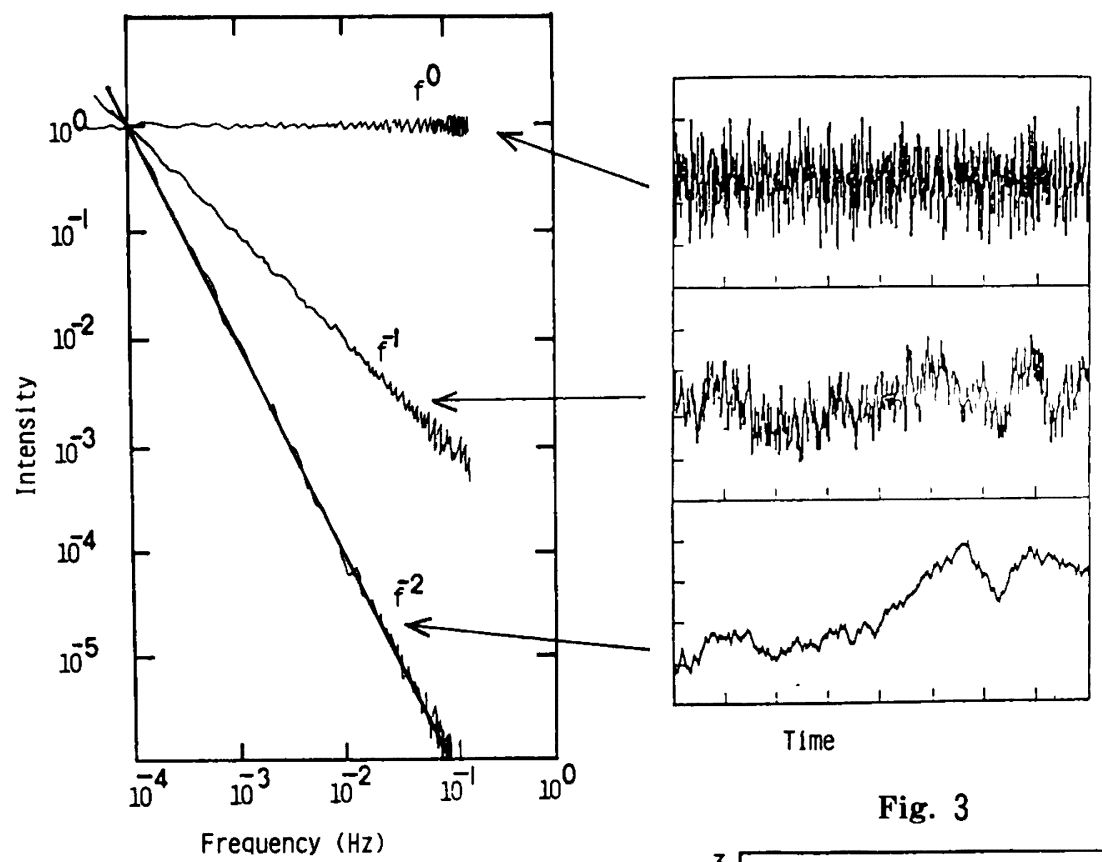

Fig. 3
Fig. 2
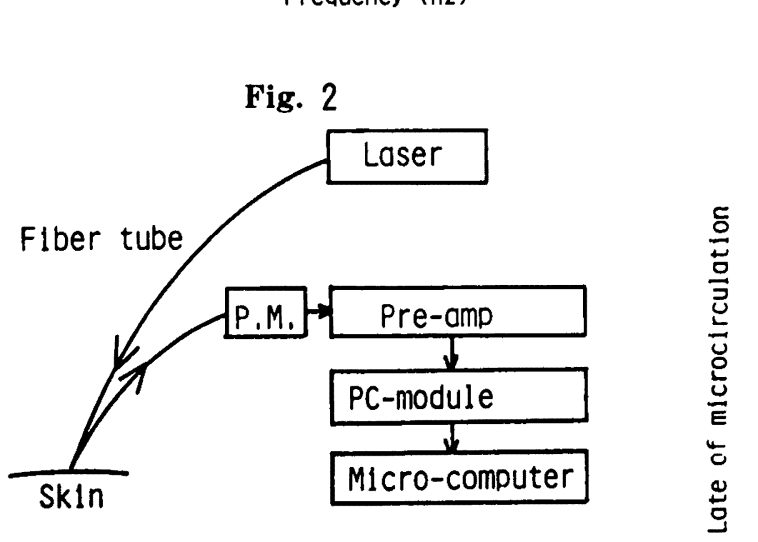

Fig. 1 Three types of spectra and their wave forms White: $f^{0}$, Pink : $f^{-1}$, Brown : $f^{-2}$

Fig. 2 Schematic diagram of measurement system

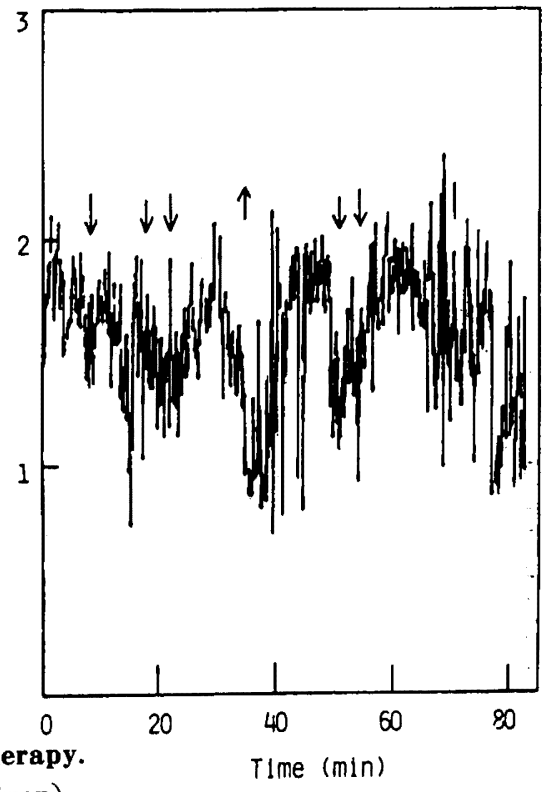

Fig. 3 The microcirculation change to a patient during therapy. (Each allow shows the time when a therapy was given) 
Fig. 4

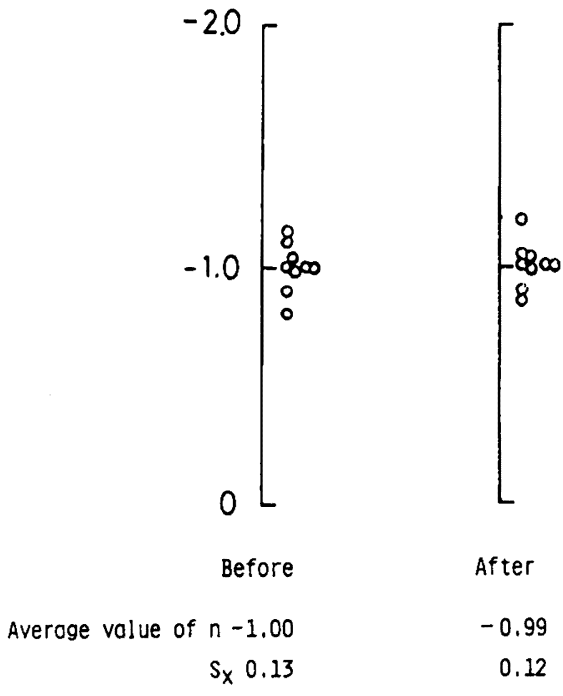

Fig. 4 Exponent numbers of $\mathrm{f}^{-\mathrm{n}}$ form of the microcirculation fluctuation for control group.

Fig. 6

(\%)

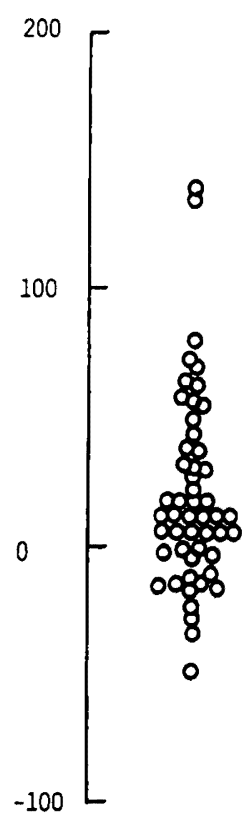

Fig. 5

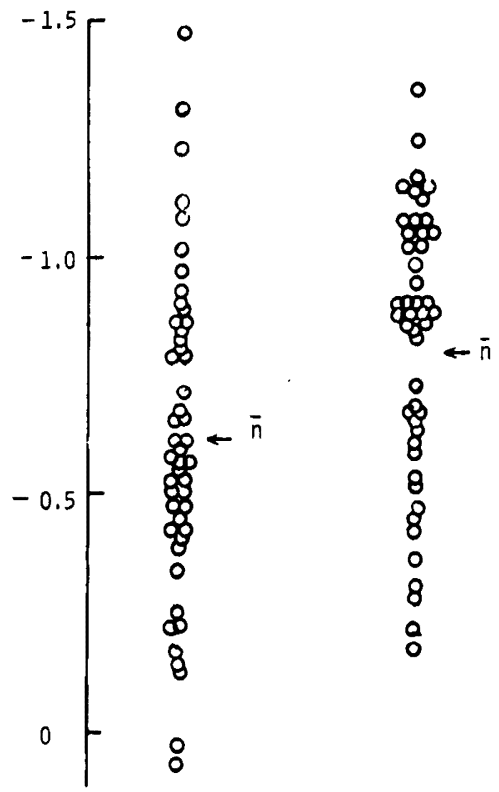

Before therapy

After theropy

Average value $n-0,611$

$-0.302$

$S_{x} \quad 0.327$

0.320

Fig. 5 Exponent numbers of $f^{-n}$ form of the microcirculation fluctuation for patients group.

Fig. 6 The changes of the late of microcirculation for patients during therapy. 